\title{
Automated Identification and B-spline Approximation of a Profiling Coil Centerline from Magnetic Resonance Images
}

\author{
S. Taivalkoski, L. Jyrkinen, and O. Silvén \\ Medical Imaging Research Group, University of Oulu, FINLAND \\ \{staivalk, 1jj\}@ee.oulu.fi
}

\begin{abstract}
Incorporation of a radiofrequency coil extending over a length of the instrument body can be used in visualising long, flexible instruments such as catheters and guidewires in magnetic resonance. Acquiring images with this RF coil results in a profile of the coil. In this work, the algorithm for an automatic identification of the coil profile centerline is presented. The algorithm consists of image segmentation and consequent instrument profile approximation with Bspline curves. The performance of the algorithm was evaluated with two profiling coil designs.
\end{abstract}

\section{Introduction}

Magnetic resonance imaging (MRI) is a well established diagnostic method, the use of which is widening toward therapeutic procedures. Some minimally invasive operations in MR guidance have been done routinely for some time now. There are, however, many new challenges that the growing interest in using MRI in therapeutic procedures has raised. These need to be faced and met, if MRI is to settle its new role as an interventional modality. A crucial problem to solve is the real-time visualisation and guidance of interventional instruments.

The localisation of rigid instruments during an operation can be done quite easily and accurately by means of optical tracking [1]. However, the localisation of flexible instruments, like catheters and guidewires, is still problematic. Passive and active tracking and profiling have been proposed for monitoring the location of flexible instruments with an MR scanner [2]. Passive methods use signal voids and susceptibility artifacts for visualising instruments. Usually, no additional scanner hardware is needed. Sometimes contrast enhancing compounds or materials strengthening susceptibility artifacts are used to improve the visibility of the instrument. Active methods use either a miniature tracking RF coil embedded in the instrument or a longer profiling coil that detects signals along the length of the instrument. While tracking of the instrument tip provides information on the location of the tip position only, profiling shows the entire instrument body which is often desirable for flexible instruments.

The identification of instruments from MR images is required by both passive and active methods. Passive methods have proven more problematic in this respect [3]. It is difficult to attain a sufficient level of contrast necessary for clearly differentiating the instrument from the background. The reason for this is that thick slices are usually 
needed to ensure that the instrument is located within the imaging volume. In thick image slices, the instrument is easily lost in the ambient tissue because of partial volume effect. Furthermore, susceptibility artifacts used for gaining more contrast often distort the images.

Active profiling avoids the problems described above. In this method, signal is received only from the immediate vicinity of the profiling coil. This removes the partial volume distortion and gives a high contrast signal, which is not dependent on slice thickness. The slice selection gradient can be switched off, leading to a projection image of the profiling coil through the entire imaging volume like in x-ray fluoroscopy. Distortions may arise due to coil geometry, noise and possible signal voids in the coil surroundings. These distortions are, however, usually less severe than susceptibility artifacts in passive profiling.

So far the major effort has been devoted to making instruments visible in MR. Less attention has been paid to visualising the instruments and utilising location information of the instruments. A common visualisation method is to extract an outline of the instrument from the MR image and to overlay it on an anatomic road map image. As Ladd et al. [4] point out, precautions must be taken to ensure that the volume acquired for the road map contains the volume of anatomy where the instrument is to be manipulated, because the instrument may lie outside the road map volume. The use of instrument location information would remove this problem, since updates of the road map could be tied to the movements of the instrument. A 3-D model of the instrument contains complete information about the location, thus offering an ideal means of controlling image acquisition and of visualising the instrument from an appropriate direction.

We have demonstrated earlier that the three dimensional geometry and location of the instrument can be reconstructed from two to three projection images [5] 6]. The identification of profiling coil centerline from MR projection images is central to the 3$\mathrm{D}$ reconstruction in our work. The centerline detection has been done semiautomatically in our former studies. Likewise, Bender et al. [7] have applied semiautomatic centerline detection for x-ray images as a part of their 3-D reconstruction algorithm. However, the need for a fully automated algorithm is evident. Semiautomatic centerline detection demands some user action to help the detection, typically selecting a starting point and a direction for the centerline. This is unacceptable if the goal is to use the algorithm in a real-time instrument guidance system.

The aim of this work has been to develop a fully automated centerline detection algorithm that is versatile and well-suited for the requirements of 3-D reconstruction. The centerline detection should be fast, and it should be able to handle images of various types of profiling coils. In addition, the algorithm should avoid any impractical restrictions of discontinuous instrument profiles and limited curvature of the centerline, which have been reported earlier [5].

\section{Methods}

\subsection{Segmentation of the Centerline}

The centerline detection algorithm can be divided into two main steps: centerline segmentation and spline fitting. The centerline detection begins with image segmentation. 
The purpose of segmentation is to extract pixels belonging to a catheter from the background of MR images. The profiling coil excites spins and receives signals from only the immediate vicinity of the catheter. Thus the major part of the background in MR images is dark due to lack of signal. The surroundings of the profiling coil appear as a bright prolonged structure.

The background noise that complicates segmentation can be estimated to follow the Rician distribution [8]. In theory, although it would be possible to find the parameters of the noise distribution from images and then remove the noise with a suitable filter, the small number of signal pixels in an image, typically just a few per cent, makes this approach unpractical. We chose to filter the background applying several filters in cascade.

In background filtering, conditional dilation is applied first. This is a standard algorithm for noise reduction when the objects to be segmented are spatially simple and connected compared to the noise. Conditional dilation thresholds the grey scale MR image with high and low threshold values. For the upper threshold, we have used a value that selects $1 \%$ of the brightest image pixels and for the lower threshold one that selects $4 \%$ of the brightest pixels. Thresholding with the high value creates a binary image in which the pixels that are binary-1 belong almost certainly to the catheter. However, a number of pixels belonging to the catheter remain binary-0 labelled. Thresholding with the lower value creates another binary image in which almost all the pixels that belong to the catheter are labelled binary-1. There are, however, many binary-1 labelled noise pixels that are misdetected and not part of the catheter. Conditional dilation fills out the high thresholded image to the bounds allowed by the lower thresholded image without including spatially disconnected noise in the result image. This produces a binary image, in which binary-1 pixels are potential catheter pixels and binary- 0 pixels belong to the background.

Small isolated groups of binary-1 pixels that still remain after conditional dilation are most probably caused by the noise. They are removed by applying erosion with a small kernel. Medial axis transformation is applied to the remaining binary-1 pixels. The purpose of this is to thin the centerline path so that only the pixels in the neighbourhood of the centerline are retained. The reduced number of pixels speeds up calculations during the following spline fitting procedure.

\subsection{Spline Fitting}

Prior to spline fitting, the segmented data points have to be ordered. The travelling salesman algorithm is used for this. The travelling salesman algorithm searches for the shortest path through the segmented data points without returning to the start point. The ordered data points form the input data set for spline fitting algorithm.

The segmented and ordered centerline data is represented in a compact mathematical form by fitting an approximating B-spline curve to the recognised data points. Bsplines are a standard way of representing curves on computers. In this context, it is possible to give only a brief summary on the topic based on [9] to illustrate the calculation of B-splines.

The B-spline curve is defined by introducing the concepts of knots and basis functions. A nondecreasing sequence of real numbers $U=\left\{u_{0}, \ldots, u_{m}\right\}$, where $u_{i} \leq$ 
$u_{i+1}, i=0, \ldots, m-1$, is called the knot vector and the $u_{i}$ are called knots. The $i$ th B-spline basis function of $p$-degree, denoted by $N_{i, p}(u)$, is defined by a recurrence formula as

$$
\begin{aligned}
& N_{i, 0}(u)= \begin{cases}1 & \text { if } u_{i} \leq u<u_{i+1} \\
0 & \text { otherwise }\end{cases} \\
& N_{i, p}(u)=\frac{u-u_{i}}{u_{i+p}-u_{i}} N_{i, p-1}(u)+\frac{u_{i+p+1}-u}{u_{i+p+1}-u_{i+1}} N_{i+1, p-1}(u) .
\end{aligned}
$$

A $p$ th degree B-spline curve is defined by

$$
\mathbf{C}(u)=\sum_{i=0}^{n} N_{i, p}(u) \mathbf{P}_{i} \quad 0 \leq u \leq 1,
$$

where the $\left\{\mathbf{P}_{i}\right\}$ are the control points, and the $\left\{N_{i, p}(u)\right\}$ are the $p$ th degree B-spline basis functions (1) defined on the knot vector

$$
U=\{\underbrace{0, \ldots, 0}_{p+1}, u_{p+1}, \ldots, u_{m-p-1}, \underbrace{1, \ldots, 1}_{p+1}\}
$$

containing $m+1$ knots. The polygon formed by the $\left\{\mathbf{P}_{i}\right\}$ is called the control polygon.

Catheter centerline is approximated to within a user-specified error bound, $E$. This error bound limits the maximum deviation between the data points and the B-spline curve to be less than $E$ everywhere on the curve. For the approximating B-spline curve, it is not usually known in advance how many control points are required to obtain the desired accuracy $E$. Thus, the approximation is an iterative process. Iteration starts with a sufficient number of control points so that the first approximation satisfies $E$. Then the number of control points is reduced and another curve is fitted. If $E$ is no longer satisfied with the new curve then the previous curve is used as the approximation curve. If $E$ is satisfied the number of control points is reduced again and iteration is repeated.

The degree of the approximating curve is elevated in steps of one until the $p$ th degree is reached. The first approximating curve is a 2nd degree curve, which is degree elevated to a 3rd degree curve. Then excess control points are discarded iteratively and the 3rd degree approximating curve is degree elevated to a 4 th degree curve. This goes on until a $p$ th degree approximating curve is reached. Working up to degree $p$ in steps decreases wriggling in the final curve and settles the curve to follow the geometric characters of the data at a proper stage. The first degree is omitted and calculation starts directly with the second degree curve, i.e. quadratic interpolation. This is justified by the smoothness of the catheter centerlines.

Fitting of the B-spline curve requires solving a linear least squares problem for the unknown control points $\mathbf{P}_{i}$. Let $n$ be the number of control points and let $\left\{\mathbf{Q}_{k}\right\}, k=$ $0, \ldots, m$ be a set of catheter points we want to approximate with a $p$ th degree B-spline curve. We assume that $p \geq 1, n \geq p$ and $m>n$. To each point $\mathbf{Q}_{k}$ we assign a precomputed parameter value $\bar{u}_{k}$. These are chosen using the chord length $d$,

$$
d=\sum_{k=1}^{m}\left|\mathbf{Q}_{k}-\mathbf{Q}_{k-1}\right|
$$


Then

$$
\begin{aligned}
& \bar{u}_{0}=0, \quad \bar{u}_{m}=1 \quad \text { and } \\
& \bar{u}_{k}=\bar{u}_{k-1}+\frac{\left|\mathbf{Q}_{k}-\mathbf{Q}_{k-1}\right|}{d} \quad k=1, \ldots, m-1 .
\end{aligned}
$$

Knots are calculated using averaging

$$
\begin{array}{rlrl}
u_{0} & =\cdots u_{p}=0, & u_{m-p}=\cdots u_{m}=1 \quad \text { and } \\
u_{j+p} & =\frac{1}{p} \sum_{i=j}^{j+p-1} \bar{u}_{i} \quad j=1, \ldots, m-2 p-1 .
\end{array}
$$

We look for the $p$ th degree B-spline curve

$$
\mathbf{C}(u)=\sum_{i=0}^{n} N_{i, p}(u) \mathbf{P}_{i} \quad u \in[0,1]
$$

satisfying $\sum_{k=0}^{m}\left|\mathbf{Q}_{k}-\mathbf{C}\left(\bar{u}_{k}\right)\right|^{2}$ is a minimum with respect to the $n+1$ variables, $\mathbf{P}_{i}$. This means that points $\mathbf{Q}_{k}$ are approximated in the least squares sense.

Let

$$
\begin{aligned}
f & =\sum_{k=0}^{m}\left|\mathbf{Q}_{k}-\mathbf{C}\left(\bar{u}_{k}\right)\right|^{2} \\
& =\sum_{k=0}^{m}\left[\left(\mathbf{Q}_{k}^{2}-2 \sum_{i=0}^{n} N_{i, p}\left(\bar{u}_{k}\right)\left(\mathbf{Q}_{k} \cdot \mathbf{P}_{i}\right)+\left(\sum_{i=0}^{n} N_{i, p}\left(\bar{u}_{k}\right) \mathbf{P}_{i}\right)^{2}\right] .\right.
\end{aligned}
$$

To minimise $f$ we set the partial derivatives of $f$ with respect to the $n$ variables, $\mathbf{P}_{l}$, equal to zero,

$$
\frac{\partial f}{\partial \mathbf{P}_{l}}=\sum_{k=0}^{m}\left(-2 N_{l, p}\left(\bar{u}_{k}\right) \mathbf{Q}_{k}+2 N_{l, p}\left(\bar{u}_{k}\right) \sum_{i=0}^{n} N_{i, p}\left(\bar{u}_{k}\right) \mathbf{P}_{i}\right)=0 .
$$

Arranging terms in (9) it follows that

$$
\sum_{i=0}^{n}\left(\sum_{k=0}^{m} N_{l, p}\left(\bar{u}_{k}\right) N_{i, p}\left(\bar{u}_{k}\right)\right) \mathbf{P}_{i}=\sum_{k=0}^{m} N_{l, p}\left(\bar{u}_{k}\right) \mathbf{Q}_{k}
$$

Equation (10) is a linear equation in the unknowns $\mathbf{P}_{0}, \ldots, \mathbf{P}_{n}$. Using (5) and (6) to compute the parameters $\left\{\bar{u}_{k}\right\}$ and the knot vector $U$ leads to a positive definite and well-conditioned coefficient matrix which has a semibandwidth less than $p+1$. Thus the equation 10 can be solved by a standard LU decomposition technique, for instance.

The deviation checking against the user-specified limit, $E$, measures maximum distance between the catheter points $\mathbf{Q}_{k}$ and the B-spline curve,

$$
\max _{0 \leq k \leq m}\left(\min _{0 \leq u \leq 1}\left|\mathbf{Q}_{k}-\mathbf{C}(u)\right|\right) \text {. }
$$

Generally

$$
\min _{0 \leq u \leq 1}\left|\mathbf{Q}_{k}-\mathbf{C}(u)\right| \leq\left|\mathbf{Q}_{k}-\mathbf{C}\left(\bar{u}_{k}\right)\right|
$$

and thus $\left\{\bar{u}_{k}\right\}$ serves as a good start point for an iterative solution of [10]. 


\section{Measurements}

We tested our centerline detection algorithm with two prototype catheters equipped with different profiling coils. A twisted pair coil was constructed by coiling a $0.1 \mathrm{~mm}$ copper wire around the outer surface of a 4 French flush catheter. Secondly, a single loop coil was embedded inside a hollow catheter. The catheter contained three channels, two of which were used as a round trip path for a $0.1 \mathrm{~mm}$ copper wire and the third was filled with $\mathrm{MnCl}_{2}$ solution. The length of both profiling coils was $40 \mathrm{~cm}$.

The profiling coil designs were visualised in a $0.23 \mathrm{~T}$ open configuration magnet manufactured by Marconi Medical Systems. Both coils were used as transmit and receive coils. During the scanning, the catheters were immersed in $0.25 \mathrm{mmol} \mathrm{MnCl}_{2}$ solution. The imaging sequences were FE-35/12 for the twisted pair coil and FE-21/8 for the single loop coil with a resolution of $128 \times 256$ without slice selection. Small 0.5 degree flip angle was used to reduce the RF transmit power to a practical level.

The profiling coils were imaged perpendicular to the main magnetic field, $B_{0}$, of the scanner. In this position, the signal to noise ratio and the apparent linewidth should be optimal for the twisted pair and suboptimal for the single loop coil [10]. We did not verify this. For the visualising purpose, we set catheters in different geometries during scanning and scanned them from axial, sagittal and coronal directions. The geometries tested were free form loop, helix and straight line.

\section{Results and Discussion}

Figures 1 and 2 show the results of the centerline detection using the projection images of catheters in different geometries as an input. It is marked on the left side of the images whether they have been taken with the twisted pair or with the single loop coil. In the series of three pictures the first one is the MR image of the profiling coil. The following two pictures show the results of segmentation and spline fitting.

The characteristic structures of the two profiling coils can be seen in these images. The twisted pair profiling coil produces a discontinuous beads-like profile of the catheter. The single loop coil has a more continuous profile, but shows a tendency to produce two parallel intense areas that are somewhat spaced from each other.

The algorithm has no difficulties in finding the centerline path of these profiles when the geometry is distinct. The algorithm is insensitive to segmentation errors in the neighbourhood of the signal intense area. A few misdetected catheter points do not disturb the correct centerline approximation. Likewise, moderate discontinuities of the segmented data are well tolerated and the spline approximation does not place restrictions on the curvature of the centerline.

On the other hand, with complex geometries like helix, the algorithm does not perform as well. In the worst cases, the centerline may not even resemble the real geometry of the coil, as it can be seen at the first three images of figure 1. The reason for this can be traced down to the travelling salesman algorithm used for arranging segmented centerline points before spline fitting. With complex geometries, the segmented data may be too complicated for travelling salesman to find the geometrically right order among the points. The shortest path is not always the correct one. 


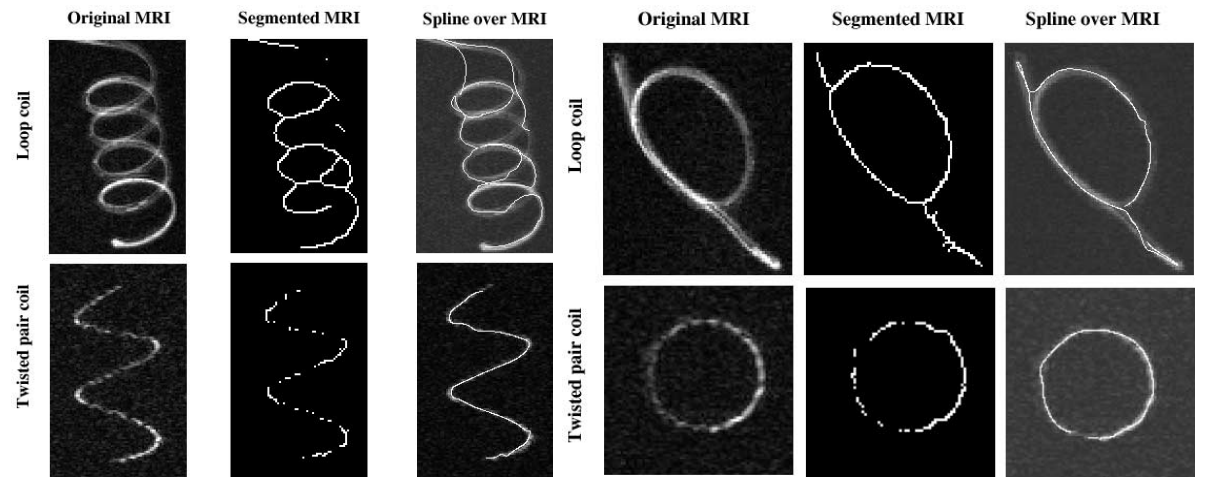

Fig. 1. Results of segmentation and spline fitting with helix and free from loop geometries
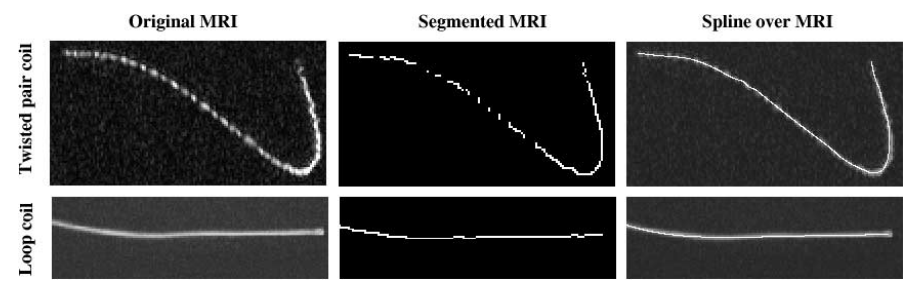

Fig. 2. Results of segmentation and spline fitting with geometries relevant to clinical applications

Failures in centerline detection may also be caused by some large intense signal areas outside the catheter profile that are missegmented to be a part of the profile. In this case, the approximating curve deviates far from the correct centerline. Fortunately, however, we found such intense noise to be relatively uncommon. If this becomes a problem, the solution is to improve the segmentation. It should be possible to find a criterion for isolated intense areas which are then excluded in the spline fitting phase.

The approximating B-spline curve is a continuous one. This causes back and forth loops in the centerline with some geometries. An example of this can be seen in the upper right corner of figure 10 Even though, it is not really a falsely detected centerline it is worth mentioning and considering, since such loops could potentially be problematic in some applications of the algorithm.

The diameter of the signal intense area is about $5 \mathrm{~mm}$. The approximated centerline stays within this intense area and thus we have a rough estimate of the maximum deviation from the real centerline path. The time needed for the calculation of the centerline varied between 0.2 and 3.7 seconds on $700 \mathrm{MHz}$ Athlon, the average time being 1.5 seconds. Compared with the image acquisition time, which was approx. 5 seconds, the image processing time is short, thus not limiting the overall performance of instrument localisation. All approximating B-spline curves are of 2nd degree. The parameter $E$ that sets the maximum allowed deviation between segmented catheter points and B-spline curve had a value $E=0.8$ in units of in-slice voxel size. 


\section{Conclusion}

We have presented an automated algorithm for the identification of a profiling coil centerline from MR images and for representing the centerline by an approximating B-spline curve. The idea has been to develop a practical tool for further higher level image processing tasks, such as instrument visualisation, and to be employed in MRI guiding tools. The need for a fully automated algorithm became evident after a number of trials with some semiautomated centerline detection algorithms.

The algorithm has been tested with two different profiling coil designs. Catheters equipped with twisted pair and single loop coils were set up and imaged in different geometries. With both coil designs, the centerlines of distinct geometries were found without problems. The algorithm tolerates discontinuities in the imaged profile of the coil and does not set restrictions for the curvature of the centerline. Complicated geometries, like helix, tend to cause incorrectly detected centerlines. However, since the geometries in real applications are usually quite simple, this failure with complex geometries cannot be considered a serious drawback.

The speed of the algorithm is reasonable. As compared with the image processing time, the image acquisition time dominates. To improve the overall performance of image acquisition and processing, the imaging time and image transfer time between the scanner and the computer should be reduced. If this is possible, we will be able to hold a promise that the algorithm now presented could serve as a base for nearly real-time instrument visualisation and guiding tools for MRI.

\section{References}

[1] Robert B. Lufkin, editor. Interventional MRI, chapter Active tracking of interventional devices, pages 144-153. MOSBY, 1999.

[2] J.F. Debatin and G. Adam, editors. Interventional Magnetic Resonance Imaging, chapter Instrument Visualization in the MR Environment. Springer, 1998.

[3] Robert B. Lufkin, editor. Interventional MRI, chapter Vascular interventional MRI, pages 387-395. MOSBY, 1999.

[4] Mark E. Ladd, Gesine G. Zimmermann, Graeme C. McKinnon, Gustav K. von Schulthess, Charles L. Dumoulin, Robert D. Darrow, Eugen Hoffmann, and Jörg F. Debatin. Visualization of vascular guidewires using MR tracking. Journal of Magnetic Resonance Imaging, 8:251-253, 1998.

[5] T.J. Vaara, J.I. Tanttu, S. Taivalkoski, and L. Jyrkinen. Catheter RF-coil profile reconstruction from 2D-projections. In Proc. of ISMRM'99, page 1953, 1999.

[6] S. Taivalkoski, L. Jyrkinen, T. Vaara, J. Tanttu, O. Tervonen, and O. Silvén. Automated catheter 3-D profile reconstruction using spline approximation. In 3rd Interventional MRI Symposium, Leipzig, May 2000.

[7] H.-J. Bender, R. Männer, C. Poliwoda, S. Roth, and M. Walz. Reconstruction of 3D catheter paths from 2D x-ray projections. In Proc of the MICCAI'99 meeting, pages 981-989, 1999.

[8] Hákon Gudbjartsson and Samuel Patz. The Rician distribution of noisy MRI data. Magnetic Resonance in Medicine, 34:910-914, 1995.

[9] Les Piegl and Wayne Tiller. The NURBS Book. Springer, 2nd edition, 1997.

[10] Mark E. Ladd, Peter Enhart, Jörg F. Debatin, Eugen Hofmann, Peter Boesiger, Gustav K. von Schulthess, and Graeme C. McKinnon. Guidewire antennas for MR fluoroscopy. Magnetic Resonance in Medicine, 37:891-897, 1997. 